\section{Sobrevida em pacientes com câncer gástrico em Campinas, São Paulo, Brasil}

\author{
Survival in gastric cancer patients \\ in Campinas, São Paulo, Brazil
}

\author{
Maria Teresa Bustamante-Teixeira 1 \\ Eduardo Faerstein 2 \\ Ângela Mariotto 3 \\ Anna Valéria de Britto 4 \\ Djalma de Carvalho Moreira Filho 4 \\ Maria do Rosário Dias de Oliveira Latorre 5
}

\title{
Introdução
}

1 Faculdade de Medicina, Universidade Federal de Juiz de Fora, Juiz de Fora, Brasil. 2 Instituto de Medicina Social, Universidade do Estado do Rio de Janeiro, Rio de Janeiro, Brasil.

3 National Cancer Institute, National Institutes of Health, Bethesda, USA. 4 Registro de Câncer de Campinas, Universidade Estadual de Campinas, Campinas, Brasil.

5 Faculdade de Saúde

Pública, Universidade de

São Paulo, São Paulo, Brasil.

Correspondência M. T. Bustamante-Teixeira Núcleo de Assessoria, Treinamento e Estudos em Saúde, Departamento de Saúde Coletiva, Faculdade de Medicina, Universidade Federal de Juiz de Fora. Rua Antônio Carlos Pereira 328, Juiz de Fora, $M G$ 36071-110, Brasil. teita@nates.ufjf.br

\section{Abstract}

This study analyzes the survival of gastric cancer patients in the city of Campinas, São Paulo State, Brazil. Data from the Campinas Population-Based Cancer Registry (RCBP) related to gastric cancer cases diagnosed from 1991 to 1994 were analyzed. Observed and relative survival rates were calculated, and to compare rates between different groups and international populations, the relative mortality risk was used. One-year relative survival rate for patients with gastric cancer was 33\%, and five-year relative survival was 9\%, confirming the poor prognosis of gastric cancer. Gender had no influence on survival, while the prognosis was better for young people. There was a gradient of severity from the localized to the metastatic state, not statistically significant. The group with undifferentiated adenocarcinomas had longer survival, with $47 \%$ of patients alive after the first year, whereas only $7 \%$ of those without a histological classification survived the first year after diagnosis. Compared with international results like the pool of European registries, the risk was greater, especially considering the five-year survival rates.

Survival Analysis; Stomach Neoplasms; Diseases Registries
O câncer de estômago vem apresentando um declínio importante de sua incidência e mortalidade nos últimos 50 anos 1,2. Apesar disto, representa ainda hoje a segunda causa de morte entre os cânceres no mundo ${ }^{3}$. No Brasil, o câncer de estômago foi a primeira causa de morte entre as neoplasias no período de 1978 a 1996. Entre os homens foi a primeira causa no período de 1979 a 1985 e entre as mulheres de 1979 a 1998, tendo sido verificada a tendência decrescente na mortalidade no período de 1977 a 19984.

O câncer de estômago apresenta taxas de incidência e mortalidade maiores nas faixas etárias mais altas e cerca de duas vezes superiores no sexo masculino 5 . Mesmo verificando-se um ligeiro aumento na sobrevida por esse câncer nos últimos anos 6,7, esta ainda é baixa. Dados europeus mostram que apenas $21 \%$ dos pacientes sobrevivem mais de cinco anos após o diagnóstico 8 .

Neste estudo, analisa-se a sobrevida dos pacientes com câncer gástrico do Município de Campinas, São Paulo, Brasil, diagnosticados entre os anos de 1991 e 1994, e compilados pelo Registro de Câncer de Base Populacional (RCBP) de Campinas. Verifica-se, ainda, o papel prognóstico de fatores, tais como o sexo e idade do paciente e a morfologia e estadiamento do tumor. Ressalte-se que o presente estudo é pio- 
neiro no Brasil, pela utilização de dados populacionais para a análise da sobrevida e pela aplicação de metodologias que permitem comparações internacionais.

O Município de Campinas apresentava, em 1996, uma população de 908.906 habitantes, correspondendo a 3\% da população do Estado de São Paulo. Com uma área de $801 \mathrm{~km}^{2}$, tratase de município eminentemente urbano, com apenas $2,7 \%$ de sua população residente em área rural. É a segunda cidade do Estado de São Paulo em termos de desenvolvimento sócioeconômico, atrás apenas da capital.

\section{Material e métodos}

Foram analisados os dados do RCBP de Campinas referentes aos casos incidentes de câncer de estômago nos anos de 1991 a 1994. Durante este período foram registrados 4.699 casos incidentes de câncer, sendo 414 de câncer de estômago identificados sob o código 151 da 9a Classificação Internacional de Doenças. Foram excluídos inicialmente da análise vinte casos notificados apenas por meio da declaração de óbito (DO), um caso com idade ignorada e um caso de carcinoma in situ.

Rotineiramente, o seguimento dos casos no RCBP de Campinas é feito de modo passivo, ou seja, confrontando-se os casos incidentes com os óbitos registrados no banco de dados do sistema de mortalidade desse município. No presente estudo considerou-se 31 de dezembro de 1999 a data final de seguimento, sendo os casos seguidos por um mínimo de cinco e um máximo de nove anos. Durante este período foram constatados 302 (77\%) óbitos entre os casos incidentes. Com base na lista dos pacientes não encontrados no sistema de mortalidade (noventa casos) foi feita uma busca ativa de informações, usando-se os prontuários médicos, nas instituições onde estes casos haviam sido identificados. Obteve-se, desta forma, informação sobre a última data de comparecimento ao serviço de $35(38,9 \%)$ desses casos. Vale salientar que, dos 35 pacientes encontrados, dez ainda estavam vivos em 31 de dezembro de 1999. Assim, considerando as informações obtidas por meio do seguimento misto, restaram 337 casos para as análises aqui apresentadas.

As variáveis independentes consideradas na análise foram: sexo, faixa etária (15-44, 4554, 55-64, 65-74 e 75+), ano de diagnóstico, estadiamento e morfologia do tumor. Para esta última variável, apesar de a literatura referir como mais indicativa de prognóstico a classificação de Lauren ${ }^{9}$, que classifica os adenocar- cinomas como do tipo intestinal e do tipo difuso, adotou-se a classificação utilizada pelo registro de câncer agrupando-as em quatro categorias principais 10 . O primeiro grupo, dito dos adenocarcinomas diferenciados, inclui todos os adenocarcinomas diferenciados no sentido intestinal, tubular, papilar e mucinoso, e ainda os adenocarcinomas sem outra especificação morfológica; um segundo grupo compreende os adenocarcinomas indiferenciados, entre os quais estão incluídos os carcinomas do tipo difuso, com células em anel de sinete, do tipo cirroso e medular; do terceiro grupo, dos não adenocarcinomas, fazem parte os tumores com metaplasia escamosa, os carcinomas adenoescamosos, epidermóides, carcinóides malignos e sarcomas; um quarto grupo reúne os tumores não especificados, que são aqueles sem exame histológico ou com exame histológico insuficiente para um diagnóstico morfológico completo.

O tempo de sobrevida foi calculado a partir da data do diagnóstico até a data do último contato ou óbito, independentemente de sua causa específica. Foram calculadas as probabilidades de sobrevida observada, utilizando-se o método atuarial $11 \mathrm{e}$, visando a eliminar o efeito da mortalidade por outras causas, calculouse a sobrevida relativa, com a utilização de programa computacional desenvolvido por Hakulinen \& Abeywickrama ${ }^{12}$. A sobrevida relativa é definida como a razão das taxas de sobrevida observada e a sobrevida geral da população onde os casos de câncer ocorreram. Para o cálculo das tábuas de vida da população, os óbitos referentes ao período 1979 a 1996 foram obtidos junto ao Ministério da Saúde, e aqueles referentes ao período 1997 a 1999 foram fornecidos pelo Sistema Municipal de Mortalidade de Campinas. As populações foram estimadas pelo método de Lagrange 13 , com base nos dados dos Censos Demográficos de 1980, 1991 e da recontagem de 1996. As taxas de sobrevida foram padronizadas por idade, tomando-se as populações padrão européia e mundial de pacientes de câncer propostas por Berrino et al. 14 e Black \& Bashir 15, respectivamente.

Visando a uma comparação mais imediata entre as taxas de sobrevida de diferentes grupos e populações internacionais, utilizou-se o risco relativo de morte, calculado como a razão do logaritmo da sobrevida relativa comparado com a categoria de referência 6,16 . Os riscos relativos de morte foram calculados para os seguintes fatores prognósticos: idade, sexo, morfologia, estadiamento e ano do diagnóstico do tumor. 


\section{Resultados}

Verificou-se uma queda acentuada dos níveis de sobrevida dos pacientes com câncer de estômago no período logo após o diagnóstico. Após o primeiro ano, a probabilidade de sobrevivência desses pacientes foi de apenas $32 \%$. O comportamento da sobrevida, ao longo dos anos, foi semelhante nos dois sexos e ao final de cinco anos a sobrevida relativa foi de $9 \%$ para ambos (Tabela 1). Entretanto, o risco relativo (RR) de morte dos homens comparado ao das mulheres foi maior, com valores estatisticamente significativos apenas na sobrevida após um ano (Tabela 2).

Em relação à idade, o prognóstico foi melhor para os indivíduos entre 45 e 54 anos, com um risco relativo de morte de 0,66 (IC95\%: 0,480,84 ) e pior para aqueles com mais de 74 anos, com um risco relativo de morte de $1,36(1,19$ 1,53), tomando-se como referência o grupo de 55-64 anos (Tabela 2). As taxas padronizadas por idade apresentaram, em geral, valores discretamente menores do que o das taxas brutas para os homens e valores discretamente maiores entre as mulheres (Tabela 1).
Considerando-se os valores da sobrevida acumulada observada e relativa em relação às variáveis analisadas (Tabela 2), observou-se uma melhora estatisticamente significativa no prognóstico desse tumor, a um ano do diagnóstico, através dos anos analisados; entretanto, aos cinco anos do diagnóstico, as taxas mais baixas referiam-se aos casos diagnosticados no ano de 1993. Verificou-se um gradiente de gravidade dos estádios localizados para aqueles mais avançados, não estatisticamente significantes. A sobrevida após o primeiro ano do diagnóstico passou de $78 \%$ entre os pacientes com tumores localizados para $16 \%$ entre aqueles com metástase a distância no momento do diagnóstico, com um risco relativo de morte de 7,38. Os pacientes para os quais esta informação não estava disponível apresentaram uma probabilidade de sobrevida no primeiro ano de $34 \%$.

Houve uma diferença na sobrevida desses pacientes de acordo com a morfologia. O grupo dos adenocarcinomas indiferenciados apresentou uma sobrevida maior, com $47 \%$ destes pacientes vivos ao final do primeiro ano, enquanto apenas $7 \%$ dos casos sem classificação

Número de casos (N), sobrevida observada e relativa (\%) e sobrevida relativa padronizada por idade (IC95\%) por câncer de estômago, segundo sexo e faixa etária. Campinas, São Paulo, Brasil, 1991 a 1994.

\begin{tabular}{|c|c|c|c|c|c|c|c|c|c|c|c|c|c|c|c|c|}
\hline & \multicolumn{12}{|c|}{ Faixa etária (anos) } & \multirow{2}{*}{\multicolumn{2}{|c|}{$\begin{array}{c}\text { ASRS } \\
\text { Européia* }\end{array}$}} & \multirow{2}{*}{\multicolumn{2}{|c|}{$\begin{array}{c}\text { ASRS } \\
\text { Mundial }^{\star \star}\end{array}$}} \\
\hline & \multicolumn{2}{|c|}{$15-44$} & \multicolumn{2}{|c|}{$45-54$} & \multicolumn{2}{|c|}{$55-64$} & \multicolumn{2}{|c|}{$65-74$} & \multicolumn{2}{|c|}{$75+$} & \multicolumn{2}{|c|}{ Total } & & & & \\
\hline & $\begin{array}{l}\text { Sobre- } \\
\text { vida } \\
\text { obser- } \\
\text { vada }\end{array}$ & $\begin{array}{l}\text { Sobre- } \\
\text { vida } \\
\text { relativa }\end{array}$ & $\begin{array}{l}\text { Sobre- } \\
\text { vida } \\
\text { obser- } \\
\text { vada }\end{array}$ & $\begin{array}{l}\text { Sobre- } \\
\text { vida } \\
\text { relativa }\end{array}$ & $\begin{array}{c}\text { Sobre- } \\
\text { vida } \\
\text { obser- } \\
\text { vada }\end{array}$ & $\begin{array}{l}\text { Sobre- } \\
\text { vida } \\
\text { relativa }\end{array}$ & $\begin{array}{l}\text { Sobre- } \\
\text { vida } \\
\text { obser- } \\
\text { vada }\end{array}$ & $\begin{array}{l}\text { Sobre- } \\
\text { vida } \\
\text { relativa }\end{array}$ & $\begin{array}{l}\text { Sobre- } \\
\text { vida } \\
\text { obser- } \\
\text { vada }\end{array}$ & $\begin{array}{l}\text { Sobre- } \\
\text { vida } \\
\text { relativa }\end{array}$ & $\begin{array}{l}\text { Sobre- } \\
\text { vida } \\
\text { obser- } \\
\text { vada }\end{array}$ & $\begin{array}{l}\text { Sobre- } \\
\text { vida } \\
\text { relativa }\end{array}$ & $\%$ & IC95\% & $\%$ & IC95\% \\
\hline Homens & \multicolumn{2}{|c|}{$(N=12)$} & \multicolumn{2}{|c|}{$(N=30)$} & \multicolumn{2}{|c|}{$(N=58)$} & \multicolumn{2}{|c|}{$(N=62)$} & \multicolumn{2}{|c|}{$(N=47)$} & \multicolumn{2}{|c|}{$(N=209)$} & & & & \\
\hline 1 ano & 20 & 21 & 45 & 47 & 37 & 39 & 28 & 29 & 19 & 20 & 30 & 32 & 29 & $22,4-35,6$ & 30 & $23,4-36,7$ \\
\hline 2 anos & 10 & 10 & 22 & 24 & 20 & 21 & 15 & 16 & 6 & 7 & 15 & 16 & 14 & $9,0-19,0$ & 15 & $9,9-20,1$ \\
\hline 3 anos & 10 & 11 & 22 & 24 & 16 & 17 & 9 & 10 & 4 & 5 & 12 & 13 & 11 & $6,7-15,3$ & 12 & $7,2-16,8$ \\
\hline 5 anos & 0 & 0 & 11 & 12 & 12 & 13 & 7 & 8 & 4 & 5 & 8 & 9 & 8 & $4,0-12,0$ & 8 & $4,3-11,7$ \\
\hline Mulheres & \multicolumn{2}{|c|}{$(N=15)$} & \multicolumn{2}{|c|}{$(\mathrm{N}=8)$} & \multicolumn{2}{|c|}{$(N=22)$} & \multicolumn{2}{|c|}{$(N=36)$} & \multicolumn{2}{|c|}{$(N=47)$} & \multicolumn{2}{|c|}{$(N=128)$} & & & & \\
\hline 1 ano & 36 & 37 & 58 & 61 & 24 & 25 & 41 & 42 & 28 & 29 & 33 & 35 & 36 & $26,9-45,1$ & 37 & $27,4-46,6$ \\
\hline 2 anos & 29 & 31 & 29 & 31 & 15 & 15 & 17 & 18 & 16 & 17 & 18 & 19 & 19 & $11,4-26,6$ & 21 & $12,6-29,4$ \\
\hline 3 anos & 9 & 10 & 29 & 31 & 10 & 10 & 17 & 18 & 7 & 7 & 12 & 13 & 13 & $6,2-19,8$ & 14 & $6,4-21,6$ \\
\hline 5 anos & 9 & 10 & 14 & 16 & 10 & 10 & 10 & 11 & 5 & 5 & 8 & 9 & 9 & $3,2-14,8$ & 10 & $3,5-16,5$ \\
\hline Total & \multicolumn{2}{|c|}{$(N=27)$} & \multicolumn{2}{|c|}{$(N=38)$} & \multicolumn{2}{|c|}{$(N=80)$} & \multicolumn{2}{|c|}{$(N=98)$} & \multicolumn{2}{|c|}{$(N=94)$} & \multicolumn{2}{|c|}{$(N=337)$} & & & & \\
\hline 1 ano & 29 & 30 & 48 & 50 & 34 & 35 & 32 & 34 & 23 & 24 & 32 & 33 & 32 & $26,7-37,3$ & 33 & $27,6-38,4$ \\
\hline 2 anos & 19 & 20 & 24 & 25 & 18 & 19 & 16 & 17 & 11 & 12 & 16 & 17 & 16 & $11,7-20,3$ & 17 & $12,5-21,5$ \\
\hline 3 anos & 10 & 10 & 24 & 25 & 14 & 15 & 12 & 13 & 6 & 6 & 12 & 13 & 12 & $8,3-15,7$ & 13 & $9,0-17,0$ \\
\hline 5 anos & 10 & 10 & 12 & 13 & 11 & 12 & 8 & 9 & 4 & 5 & 8 & 9 & 9 & $5,7-12,3$ & 9 & $5,5-12,5$ \\
\hline
\end{tabular}

* Taxas padronizadas por idade segundo população padrão européia;

** Taxas padronizadas por idade segundo população padrão mundial. 
Número de casos, sobrevida observada e relativa (\%) de pacientes com câncer de estômago a 1 e 5 anos do diagnóstico e risco relativo (RR) segundo variáveis analisadas. Campinas, São Paulo, Brasil, casos diagnosticados entre 1991 e 1994.

\begin{tabular}{|c|c|c|c|c|c|c|c|c|c|c|c|}
\hline & \multirow{3}{*}{$\begin{array}{l}\text { Número } \\
\text { de casos }\end{array}$} & \multicolumn{6}{|c|}{ Probabilidade de sobrevida (\%) } & \multirow{2}{*}{\multicolumn{2}{|c|}{1 ano }} & \multirow{2}{*}{\multicolumn{2}{|c|}{5 anos }} \\
\hline & & 1 ar & & IC95\% & 5 an & & IC95\% & & & & \\
\hline & & Observada & Relativa & & Observada & Relativa & & $\mathrm{RR}$ & IC95\% & $\mathrm{RR}$ & IC95\% \\
\hline \multicolumn{12}{|l|}{ Sexo } \\
\hline Feminino & 128 & 33 & 35 & $34,9-35,1$ & 8 & 9 & $8,9-9,1$ & 1 & & 1 & Referência \\
\hline Masculino & 209 & 30 & 32 & $31,9-32,1$ & 8 & 9 & $8,9-9,0$ & 1,09 & $1,00-1,17$ & 1 & $0,97-1,03$ \\
\hline \multicolumn{12}{|l|}{ Grupo etário } \\
\hline $15-44$ & 27 & 29 & 30 & $29,8-30,2$ & 10 & 10 & $9,9-10,1$ & 1,15 & $0,93-1,36$ & 1,09 & $1,01-1,16$ \\
\hline $45-54$ & 38 & 48 & 50 & $49,8-50,2$ & 12 & 13 & $12,9-13,1$ & 0,66 & $0,48-0,84$ & 0,96 & $0,90-1,03$ \\
\hline $55-64$ & 80 & 34 & 35 & $34,9-35,1$ & 11 & 12 & $11,9-12,1$ & 1,00 & Referência & 1,00 & Referência \\
\hline $65-74$ & 98 & 32 & 34 & $33,9-34,1$ & 8 & 9 & $8,9-9,1$ & 1,03 & $0,88-1,17$ & 1,14 & $1,08-1,19$ \\
\hline$>74$ & 94 & 23 & 24 & $23,9-24,1$ & 4 & 5 & $4,9-5,1$ & 1,36 & $1,19-1,53$ & 1,41 & $1,36-1,47$ \\
\hline \multicolumn{12}{|l|}{ Ano do diagnóstico } \\
\hline 1991 & 106 & 24 & 24 & $23,9-24,1$ & 10 & 10 & $9,9-10,1$ & 1,00 & Referência & 1,00 & Referência \\
\hline 1992 & 103 & 29 & 30 & $29,9-30,1$ & 8 & 8 & $7,9-8,1$ & 0,84 & $0,76-0,93$ & 1,10 & $1,06-1,14$ \\
\hline 1993 & 66 & 37 & 38 & $37,9-38,1$ & 2 & 2 & $1,9-2,0$ & 0,68 & $0,58-0,77$ & 1,70 & $1,65-1,75$ \\
\hline 1994 & 62 & 44 & 49 & $48,9-49,1$ & 13 & 14 & $13,9-14,1$ & 0,50 & $0,40-0,60$ & 0,85 & $0,81-0,90$ \\
\hline \multicolumn{12}{|l|}{ Estádio } \\
\hline Localizado & 8 & 73 & 78 & $77,7-78,4$ & 73 & 81 & $80,6-81,4$ & 1,00 & Referência & 1,00 & $-1,34-3,34$ \\
\hline Regional & 47 & 39 & 41 & $40,9-41,2$ & 15 & 17 & $16,9-17,1$ & 3,59 & $-1,34-8,51$ & 8,41 & $-5,54-22,35$ \\
\hline Metástase a distância & 54 & 15 & 16 & $15,9-16,1$ & 0 & 0 & & 7,38 & $-2,68-17,43$ & - & \\
\hline Ignorado & 228 & 33 & 34 & $33,9-34,1$ & 7 & 7 & $6,9-7,0$ & 4,34 & $-1,58-10,26$ & 12,62 & $-8,29-33,53$ \\
\hline \multicolumn{12}{|l|}{ Morfologia ${ }^{\star \star}$} \\
\hline $\begin{array}{l}\text { Adenocarcinoma } \\
\text { diferenciado }\end{array}$ & 235 & 33 & 35 & $34,9-35,1$ & 9 & 10 & $9,9-10,0$ & 1,00 & Referência & 1,00 & Referência \\
\hline $\begin{array}{l}\text { Adenocarcinoma } \\
\text { não diferenciado }\end{array}$ & 56 & 45 & 47 & $46,9-47,1$ & 10 & 11 & $10,9-11,1$ & 0,72 & $0,58-0,86$ & 0,96 & $0,92-1,00$ \\
\hline Não adenocarcinoma & 4 & 25 & 26 & $25,6-26,5$ & 0 & 0 & & 1,28 & $0,86-1,71$ & - & \\
\hline Não especificado & 42 & 7 & 7 & $6,9-7,1$ & 2 & 3 & $2,9-3,0$ & 2,53 & $2,36-2,71$ & 1,52 & $1,49-1,56$ \\
\hline Total & 337 & 32 & 33 & $32,9-33,1$ & 8 & 9 & $8,9-9,0$ & & & & \\
\hline
\end{tabular}

histológica sobreviveram ao primeiro ano após o diagnóstico. Tomando como referência os tumores classificados no grupo dos adenocarcinomas diferenciados, o risco relativo de morte foi significativamente menor para os adenocarcinomas indiferenciados com valores de 0,72 (IC95\%: 0,58-0,86) e 0,96 (IC95\%: 0,92-1,00) a 1 e 5 anos do diagnóstico, respectivamente, enquanto que aqueles tumores com morfologia não especificada apresentaram riscos relativos de morte 2,53 (IC95\%: 2,36-2,71) e 1,52 (IC95\%: $1,49-1,56)$ vezes maiores quando comparados ao mesmo grupo, a 1 e 5 anos do diagnóstico.

\section{Discussão}

Este estudo analisa a sobrevida dos pacientes com câncer de estômago residentes no Município de Campinas, coletados pelo RCBP de Campinas nos anos de 1991 a 1994, constituindo-se, até onde se sabe, no primeiro estudo deste gênero no Brasil.

Comparando-se com dados europeus apresentados pelo projeto EUROCARE 6, a incidência de câncer gástrico em Campinas ocorreu em uma população mais jovem, sendo o percentual de maiores de 74 anos de $35 \%$ e $27 \%$, respectivamente para os registros europeus e de Campinas, com maior predominância masculina $(60 \%$ 
e 55\%, respectivamente). Quanto à qualidade dos dados, o percentual de casos registrados com base apenas na DO, em Campinas, foi de $5 \%$, menor do que a média européia de $7 \%$. Em 89\% dos casos houve confirmação microscópica, índice superior aos $78 \%$ verificados nos registros europeus. A proporção de casos perdidos de seguimento no registro de Campinas foi de $14 \%$, bem maior do que a observada nos registros europeus, que foi de $0,2 \%$, mas comparável à dos registros de países em desenvolvimento 17 .

Os valores de $33 \%$ e $9 \%$ de sobrevida relativa após 1 e 5 anos de diagnóstico, respectivamente, encontrados em Campinas, confirmam, ainda uma vez, o mau prognóstico dos pacientes com câncer de estômago. Mesmo na Áustria, país que apresenta os melhores resultados europeus, $51 \%$ desses pacientes sobrevivem após um ano do diagnóstico, enquanto que apenas $27 \%$ alcançam uma sobrevida de cinco anos. Nos Estados Unidos, o programa SEER refere uma taxa de sobrevida relativa aos cinco anos de $18 \%$ para os pacientes brancos 18 , valores muito próximos aos da média européia de $21 \%$. Resultados melhores são observados em Osaka, Japão, onde a taxa de sobrevida relativa aos cinco anos foi de $47 \%$, demonstrando os resultados dos esforços dos últimos trinta anos no combate a essa doença no Japão, principalmente por meio do diagnóstico precoce 19.

Comparando a taxas de sobrevida relativa após cinco anos do diagnóstico padronizadas por idade, dos países em desenvolvimento 17, constatam-se valores semelhantes para os registros de Chennai na Índia (ASRS\% 5 anos $=7,8$ ), Chiang Mai na Tailândia $(7,9)$ e Rizal nas Filipinas $(9,6)$. Entretanto, os registros de Qidong $(13,0)$ e Shangai $(22,7)$ na China, e Khon Kaen $(23,1)$ na Tailândia, apresentam valores superiores aos 9\% encontrados em Campinas.

As taxas encontradas em Campinas foram comparadas às dos países europeus (Tabela 3) e revelaram um risco de morte $24 \%$ e $54 \%$ maior no primeiro e no quinto ano após o diagnóstico, assemelhando-se aos resultados observados na Dinamarca, Eslovênia, Eslováquia e Estônia 10 . Observou-se que a razão de taxas revela-se maior quando comparadas às taxas de sobrevida aos cinco anos nos diversos países, enquanto a diferença entre os riscos de morte a 1 e aos 5 anos permanece semelhante entre os países analisados, mantendo-se praticamente constante em um mesmo país. Apenas para Campinas (Tabela 3), observa-se que o risco relativo aos cinco anos foi maior do que a um ano, o que poderia ser atribuído a diferenças no estadiamento da doença ao diagnóstico ou ao tratamento realizado.
Cabe ressaltar, que em relação ao câncer gástrico, considerado como um dos tumores de mau prognóstico, assim como os de fígado, pâncreas, pulmão e esôfago, as diferenças de sobrevida verificadas entre os países desenvolvidos e aqueles em desenvolvimento são reduzidas. Estas diferenças são mais acentuadas para tumores como os de cabeça e pescoço, cólon e mama, que podem ter seu prognóstico substancialmente melhorado se detectados precocemente e tratados adequadamente 17.

Algumas dessas diferenças encontradas na sobrevida podem ser atribuídas a diferenças metodológicas de difícil controle no registro e no seguimento dos casos. Os controles propostos e realizados pelo programa EUROCARE, que foram aplicados também aos dados de Campinas, sugerem que os problemas metodológicos tenham sido controlados, permitindo a comparação. Nos registros de câncer, uma questão metodológica importante é a do seguimento dos casos, especialmente para os registros dos países em desenvolvimento. Neste estudo, optou-se pelo o seguimento misto, que tende a gerar taxas de sobrevida mais conservadoras.

As mulheres geralmente apresentam uma expectativa maior de vida e vários estudos têm sugerido que esta vantagem também é verificada nas pacientes com câncer 14,20,21. No presente estudo, esta variação entre os sexos foi observada apenas na sobrevida após um ano do diagnóstico $\left(\mathrm{RR}_{1 \mathrm{ano}}=1,09\right)$ (Tabela 3$)$. Micheli et al. 16 encontraram um RR de 0,9 para os registros europeus, demonstrando esta pequena vantagem na sobrevida por câncer de estômago para o sexo feminino. Em relação à idade, os pacientes entre 45 e 54 anos obtiveram maiores taxas de sobrevida quando comparadas às demais. Estes resultados diferem dos relatados pelos registros europeus por meio do projeto EUROCARE II 6 e pelos registros italianos 10 , que mostram uma sobrevida maior para o grupo etário mais jovem (15-44 anos). No entanto, confirma-se, nesses estudos, o padrão de risco elevando-se com o aumento da idade.

Quanto à morfologia, comparando-se os achados de Campinas com os dos registros italianos que adotam esta mesma classificação, confirma-se o risco significativamente maior de morte para os tumores sem classificação morfológica em relação aos adenocarcinomas diferenciados. Não verificou-se, porém, o risco maior dos adenocarcinomas indiferenciados em relação aos adenocarcinomas diferenciados constatado pelos dados italianos 10 .

Verdecchia et al. 22,23, comparando taxas de incidência e de sobrevida de diferentes países, observaram que a sobrevida por câncer gástri- 
Tabela 3

Número de casos, taxas de sobrevida padronizadas por idade (ASRS\%) após 1 e 5 anos do diagnóstico de câncer de estômago em diversos países.

\begin{tabular}{|c|c|c|c|c|c|c|c|c|c|c|c|}
\hline Registro & Período & $\begin{array}{l}\text { Número } \\
\text { de casos }\end{array}$ & $\begin{array}{l}\text { Idade } \\
\text { (média) }\end{array}$ & $\begin{array}{l}\text { ASRS\% } \\
\text { (1 ano) }\end{array}$ & IC95\% & $\mathrm{RR}^{\star}$ & $\begin{array}{c}\text { IC } \\
\text { (RR95\%) }\end{array}$ & $\begin{array}{l}\text { ASRS\% } \\
\text { (5 anos) }\end{array}$ & IC95\% & $\mathrm{RR}^{*}$ & $\begin{array}{c}\text { IC } \\
\text { (RR95\%) }\end{array}$ \\
\hline Campinas & 1991-1994 & 337 & 65,5 & 32 & $27-37$ & 1,24 & $1,06-1,43$ & 9 & $6-12$ & 1,54 & $1,31-1,78$ \\
\hline Europa (pool) & 1985-1989 & 64.064 & 65,5 & 40 & $39-41$ & Referência & & 21 & $20-22$ & Referência & \\
\hline Islândia & 1985-1989 & 250 & 69,9 & 45 & $38-51$ & 0,87 & $0,71-1,03$ & 23 & $18-30$ & 0,94 & $0,78-1,11$ \\
\hline Finlândia & 1985-1989 & 2.539 & 69,2 & 40 & $38-41$ & 1,00 & $0,98-1,05$ & 20 & $18-21$ & 1,03 & $0,97-1,09$ \\
\hline Suécia & 1985-1989 & 1.276 & 72,0 & 41 & $38-44$ & 0,97 & $0,89-1,06$ & 17 & $15-20$ & 1,14 & $1,04-1,23$ \\
\hline Dinamarca & 1985-1989 & 3.545 & 70,6 & 31 & $29-33$ & 1,28 & $1,20-1,36$ & 13 & $12-15$ & 1,31 & $1,23-1,39$ \\
\hline Escócia & 1985-1989 & 5.597 & 70,4 & 27 & $26-29$ & 1,43 & $1,36-1,50$ & 11 & $10-12$ & 1,41 & $1,34-1,49$ \\
\hline Inglaterra & 1985-1989 & 23.292 & 71,3 & 28 & $27-29$ & 1,39 & $1,34-1,44$ & 12 & $11-12$ & 1,36 & $1,31-1,41$ \\
\hline Holanda & 1985-1989 & 1.026 & 69,5 & 40 & $37-43$ & 1,00 & $0,91-1,09$ & 19 & $17-23$ & 1,06 & $0,96-1,17$ \\
\hline Alemanha & 1985-1989 & 1.307 & 69,1 & 43 & $40-46$ & 0,92 & $0,84-1,09$ & 26 & $23-29$ & 0,86 & $0,78-0,94$ \\
\hline Áustria & 1988-1989 & 362 & 70,5 & 51 & $45-56$ & 0,73 & $0,59-0,83$ & 27 & $22-33$ & 0,84 & $0,71-0,97$ \\
\hline Suiç̧a & 1985-1989 & 555 & 69,9 & 43 & $40-47$ & 0,92 & $0,83-1,01$ & 23 & $19-28$ & 0,94 & $0,81-1,07$ \\
\hline França & 1985-1989 & 1.479 & 70,9 & 48 & $45-50$ & 0,80 & $0,74-0,86$ & 25 & $22-28$ & 0,89 & $0,81-0,97$ \\
\hline Espanha & 1985-1989 & 3.015 & 67,0 & 43 & $41-55$ & 0,92 & $0,86-0,98$ & 26 & $24-28$ & 0,86 & $0,81-0,92$ \\
\hline Itália & 1985-1989 & 8.518 & 70,3 & 44 & $43-45$ & 0,90 & $0,86-0,93$ & 23 & $22-24$ & 0,94 & $0,90-0,98$ \\
\hline Eslovênia & 1985-1989 & 2.383 & 66,2 & 31 & $29-33$ & 1,28 & $1,20-1,36$ & 14 & $9-22$ & 1,26 & $0,97-1,55$ \\
\hline Eslováquia & 1985-1989 & 5.201 & 66,4 & 33 & $32-34$ & 1,21 & $1,16-1,26$ & 19 & $17-20$ & 1,06 & $1,00-1,13$ \\
\hline Polônia & 1985-1989 & 1.180 & 67,4 & 24 & $22-27$ & 1,56 & $1,44-1,68$ & 9 & $7-11$ & 1,54 & $1,39-1,70$ \\
\hline Estônia & 1985-1989 & 2.539 & 64,1 & 33 & $31-35$ & 1,21 & $1,14-1,28$ & 16 & $14-18$ & 1,17 & $1,09-1,26$ \\
\hline
\end{tabular}

* Risco relativo de morte de cada país ou registro versus Europa.

co é maior em áreas de maior incidência. Constataram que o estadiamento foi o fator mais importante para explicar a variabilidade desta sobrevida, acrescida da idade, tipo histológico e da sublocalização do tumor.

É amplamente aceito que o mais importante fator prognóstico para a sobrevida por câncer de estômago é o estadiamento do tumor ao diagnóstico, pois isto determina o tratamento, principalmente se este tumor é cirúrgico. Diferenças de sobrevida encontradas entre as diversas populações analisadas podem ser atribuídas a este fator 6 . No caso de Campinas, o estádio do tumor ao diagnóstico se apresenta como um importante fator prognóstico, apesar de esta informação estar disponível apenas para $32,3 \%$ dos casos analisados.

As limitações deste estudo são inerentes àquelas ligadas à utilização de dados secundários. No entanto, para os RCBP são bem descritos normas e procedimentos 24 adotados mundialmente, o que permite uma qualidade adequada e a comparabilidade dos resultados. Algumas variáveis, como o estadiamento, estão disponíveis apenas para um pequeno número de observações, gerando estimativas menos precisas. No entanto, analisar os dados produ- zidos é, seguramente, a melhor forma de estimular esses RCBP a gerarem informações com qualidade cada vez melhor.

As taxas de sobrevida para câncer gástrico têm mostrado um discreto aumento nos últimos anos na Europa 8 e um aumento expressivo no Japão ${ }^{19}$. Neste trabalho, não foi possível avaliar estas tendências devido ao pequeno número de anos disponíveis no RCBP de Campinas.

A redução da mortalidade e o aumento da sobrevida de câncer de estômago por meio da detecção precoce é limitada, devido ao alto custo da radiografia fotofluográfica e da gastroscopia subseqüente indicada para cerca de $10 \%$ dos indivíduos examinados, devendo ser indicada para as regiões de alto risco. A estratégia de controle desse tumor mais viável a longo prazo, e indicada para as regiões de menor risco, é a da prevenção primária, centrada principalmente no estímulo a uma dieta adequada 25 . 


\section{Resumo}

Neste estudo, analisa-se a sobrevida de pacientes diagnosticados com câncer gástrico no Município de Campinas, São Paulo, Brasil. Foram analisados os dados do Registro de Câncer de Base Populacional (RCBP) de Campinas referentes aos casos incidentes nos anos de 1991 a 1994. Calculou-se a sobrevida observada e relativa e, visando a uma comparação entre as taxas de sobrevida de diferentes grupos e populações internacionais, utilizou-se o risco relativo de morte. A sobrevida relativa foi de $33 \%$ e $9 \%$ ao final do primeiro e do quinto ano após o diagnóstico, respectivamente. Não foi constatada diferença na sobrevida por câncer de estômago entre os sexos; o prognóstico revelou-se melhor para os indivíduos mais jovens. Verificou-se um gradiente de gravidade dos estádios localizados para aqueles mais avançados, não estatisticamente significantes. O grupo dos adenocarcinomas indiferenciados apresentou uma sobrevida maior, com $47 \%$ destes pacientes vivos ao final do primeiro ano, enquanto apenas 7\% dos casos sem classificação histológica sobreviveu ao primeiro ano após o diagnóstico. Comparando-se com resultados internacionais, tais como os de registros europeus, Campinas revelou um risco de morte maior do que o da média dos registros europeus, especialmente considerando-se o quinto ano após o diagnóstico.

Análise de Sobrevivência; Neoplasias Gástricas; Registros de Doenças

\section{Colaboradores}

A. V. Britto e D. C. Moreira Filho foram responsáveis pela coleta, codificação e armazenamento dos dados. M. T. Bustamante-Teixeira realizou a revisão do banco de dados e do seguimento dos casos, a revisão bibliográfica, a análise dos dados e a redação do artigo. E. Faerstein, A. Mariotto e M. R. D. O. Latorre contribuíram na análise dos dados e na revisão do artigo.

\section{Agradecimentos}

Nossos agradecimentos a Arduino Verdechia, Ricardo Capocaccia e Gianni De Angelis e aos colegas do Istituto Superiore di Sanità, de Roma, Itália, pelo apoio técnico e acolhida carinhosa durante o desenvolvimento da pesquisa. Nossos agradecimentos também a Nazyra Mahayri, atual coordenadora do Registro de Câncer de Base Populacional (RCBP) de Campinas, a Sônia Duarte pelo apoio na coleta dos dados do seguimento dos pacientes, e a Marilisa Berti e Letícia de Las Mercedes Marin Leon, da Universidade Estadual de Campinas (UNICAMP), por disponibilizarem prontamente os dados de mortalidade de Campinas. Agradecemos ainda a todos os hospitais, instituições e pessoas que colaboram com o RCBP de Campinas.

\section{Referências}

1. Kurihara M, Aoki K, Tominaga S, editors. Cancer mortality statistics in the world. Nagoya: University of Nagoya Press; 1984.

2. Parkin DM, Pisani P, Ferlay J. Estimates of the world-wide incidence of 25 major cancers in 1990. Int J Cancer 1999; 80:827-41.

3. Pisani P, Parkin DM, Bray F, Ferlay J. Estimates of the worldwide mortality from 25 cancers in 1990 . Int J Cancer 1999; 83:870-3.

4. Latorre MRDO. A mortalidade por câncer de estômago no Brasil: análise do período de 1977 a 1989. Cad Saúde Pública 1997; 13 Suppl 1:67-78.

5. Parkin DM, Whelan SL, Ferlay J, Raymond L, Young J, editors. Cancer incidence in five continents. v. VII. Lyon: International Agency for Research on Cancer; 1997. (IARC Scientific Publications, 143).

6. Faivre J, Forman D, Estéve J, Gatta G; EUROCARE Working Group. Survival of patients with oesophageal and gastric cancers in Europe. Eur J Cancer 1998; 14:2167-75.

7. Miller BA, Ries LA, Hankin BF, Kosary CI, Harras A, Devesa BK, editors. SEER cancer statistics review: 1973-1990. Bethesda: National Institutes of Health; 1993. (NIH Publication, 93).

8. Berrino F, Capocaccia R, Estève J, Gatta G, Hakulinen T, Micheli A, et al., editors. Survival of cancer patients in Europe: The EUROCARE-2 study. Lyon: International Agency for Research on Cancer; 1999. (IARC Scientific Publications, 151).

9. Lauren P. The two histologycal main types of gastric carcinoma: diffuse and so-called intestinal-type carcinoma. Acta Pathol Microbiol Scand 1965; 64:31-49.

10. Gatta G, Sant M, Micheli A, Capocaccia R, Verdecchia A, Barchielli A, et al. La sopravvivenza per tumori dell'apparato digerente: dati italiani su base di popolazione e confronti internaziolnali. Ann Ist Super Sanita 1996; 32:513-25.

11. Lee ET. Statistical methods for survival analysis. 2nd Ed. New York: John Wiley \& Sons; 1992.

12. Hakulinen T, Abeywickrama KH. A computer program package for relative survival analysis. Comput Programs Biomed 1985; 19:197-207.

13. Cláudio DM, Marins JM. Cálculo numérico computacional. São Paulo: Editora Atlas; 1989.

14. Berrino F, Sant M, Verdecchia A, Capocaccia R, Hahulinen T, Estève J, editors. Survival of cancer patients in Europe: The EUROCARE study. Lyon: International Agency for Research on Cancer; 1995. (IARC Scientific Publications, 132).

15. Black RJ, Bashir SAR. World standard cancer patient populations: a resource for comparative analysis of survival data. In: Sankaranarayanan R, Black RJ, Parkin DM, editors. Cancer survival in developing countries. Lyon: International Agency for Research on Cancer; 1998. p. 9-12. (IARC Scientific Publications, 145).

16. Micheli A, Mariotto A, Giorgi-Rossi A, Gatta G, Muti P; EUROCARE Working Group. The prognostic role of gender in survival of adult cancer patients. Eur J Cancer 1998; 14:2271-8.

17. Sankaranarayanan R, Black RJ, Parkin DM. Cancer survival in developing countries. Lyon: International Agency for Research on Cancer; 1998. (IARC Scientific Publications, 145). 
18. Kosary CL, Ries LAG, Miller BA, Mankey BF, Harras A, Edwards BK. SEER cancer statistics review 1973-1992. Bethesda: National Institutes of Health; 1995. (NIH Publication, 96).

19. Hisamichi S, Sugawara N. Mass screening for gastric cancer by X-ray examination. Jpn J Clin Oncol 1984; 14:211-23.

20. Adami HO, Bergström R, Klareskog L, Persson I, Pontén J. The effect of female sex hormone on cancer survival. JAMA 1990; 263:2189-93.

21. Gatta G, Buiatte E, Conti E, De Lisi V, Falcini F, Federico M, et al. Variations in the survival of adult cancer patients in Italy. Tumori 1997; 83: 497-504.

22. Verdecchia A, Corazziari I, Gatta G, Lisi D, Faivre J, Forman D, et al. Explaining gastric cancer survival differences among European countries. Int J Cancer 2004; 109:737-41.
23. Verdecchia A, Mariotto A, Gatta G, BustamanteTeixeira MT, Ajiki W. Comparison of stomach cancer incidence and survival in four continents. Eur J Cancer 2003; 39:1603-9.

24. Jensen OM, Parkin DM, MacLennan R, Muir CS, Skeet RG. Cancer registration principles and methods. Lyon: International Agency for Research on Cancer; 1991. (IARC Scientific Publications, 95).

25. Nomura A. Stomach cancer. In: Schottenfeld D, Fraumeni Jr. JF, editors. Cancer epidemiology and prevention. New York; Oxford University Press; 1996. p. 707-24.

Recebido em 21/Jun/2005

Versão final reapresentada em 04/Nov/2005 Aprovado em 28/Nov/2005 